\title{
Magnitude of Hepatitis B Virus and Hepatitis C Virus among HAART Taking Patients and Association with Liver and Renal Function and CD4+ T Cells Level
}

Nebiyou Yemanebrhane $^{1 *}$, Desalegn Addise ${ }^{2}$, Nardos Abebe ${ }^{2}$, Fassika Abebe ${ }^{3}$, Aster Shewaamare ${ }^{4}$ and Aster Tsegaye ${ }^{5}$

${ }^{1}$ Bethel Teaching General Hospital, Addis Ababa, Ethiopia

${ }^{2}$ Ethiopian Public Health Institute, Addis Ababa, Ethiopia

${ }^{3}$ Beker Pharmaceuticals, Addis Ababa, Ethiopia

${ }^{4}$ Zewditu Memorial Hospital, Addis Ababa, Ethiopia

${ }^{5}$ Addis Ababa University, College of Health Science, Department of Medical Laboratory Sciences, Addis Ababa, Ethiopia

\begin{abstract}
Background: Co-infection of Hepatitis B virus (HBV) and Hepatitis C virus (HCV) are very prevalent and the primary cause for morbidity and mortality among patients with Human immunodeficiency Virus (HIV). The aim of this study was to assess the magnitude of HBV and HCV infection and its association with liver and renal function, and CD4+ T cell levels of highly active anti-retroviral therapy (HAART) taking patients.
\end{abstract}

Method: A hospital based cross sectional study was conducted at Zewditu Memorial Hospital. Participants' sociodemographic and clinical information were collected from the anti-retroviral therapy (ART) database. Blood samples were collected from 384 volunteer, patients taking HAART for at least 1 year to investigate CD4, HBSAg, HCVAb, liver and renal function. Data was entered and analyzed by SPSS version 20 and $p$ value less than 0.05 was taken as statistically significant.

Results: Of the 384 participants, $222(57.81 \%)$ were females, and $168(43.75 \%)$ were in age group 30-39 years. Overall burden of HBV and HCV were 21 (5.7\%). Co-infection rates of HBV-HIV, HCV-HIV and HBV-HCV-HIV were 18 $(4.7 \%), 4(1 \%)$ and $1(0.2 \%)$, respectively. Co-infected study participants had significantly raised mean liver enzyme levels (AST, ALT and ALP) than mono-infected ones. There was no statistically significant difference for renal function parameters between mono-infected and co-infected HAART taking patients. Co-infected HIV patients had lower mean level of CD4 compared to mono-infected ones.

Conclusion: HBV and HCV co-infected HIV patients are more likely to have abnormal liver enzyme levels and tend to have low mean CD4 count than mono-infected HIV patients. Large scale cohort study is recommended for consolidating the current findings.

Keywords: HIV; HBV; HCV; CD4 count; Liver function; Renal function; Co-infection

\section{Introduction}

Hepatitis B virus (HBV) is a member of the Hepadnaviridae family, a small deoxy ribonucleic acid (DNA) virus, which replicates through a ribonucleic acid (RNA) intermediate and can integrate into the host genome [1]. These virus is a major risk factor for hepatocellular carcinoma [2]. The hepatitis $\mathrm{C}$ virus (HCV) is a spheric, enveloped RNA virus of the Flaviviridae family. HCV has been recognized as a major cause of chronic hepatitis and hepatic fibrosis that progresses in some patients to cirrhosis and hepatocellular carcinoma [3]. Human immunodeficiency Virus (HIV) is RNA virus which belongs to the family of lentiviruses, slow replicating virus. It remains a major global challenge especially in sub Saharan African countries $[4,5]$.

Globally, HBV, HCV and HIV account for an estimated 350-370, 130-170 and 40 million chronic infections, respectively [6-8]. Recent study by Belyhun et al. indicated that overall pooled prevalence of HBV and HCV in Ethiopia are 7.4\% and 3.1\%, respectively [9]. In subSaharan Africa, the overall prevalence of HBV and HCV co-infection in HIV infected people were $15 \%$ and $7 \%$, respectively [10]. In Ethiopia, 1.3 million people live with HIV (78\% adults) [11]. Study conducted in north-west Gondar showed that overall prevalence of viral hepatitis among HIV patients was $11.7 \%$. The seroprevalence of HBV-HIV and HCV-HIV co-infection were $5.6 \%$ and 5.0\%, respectively [12].

Because they share similar transmission routes, HBV and HCV are very prevalent and among the main reasons of morbidity and mortality in HIV patients. Co-infection of HBV or HCV with HIV has been associated with reduced survival, increased risk of progression to liver disease, and increased risk of hepatotoxicity, associated with antiretroviral therapy $[13,14]$. In addition, the gains of HAART also compromised by co-infection with hepatitis viruses as they are known to have adverse effects on the prognosis of HIV and hepatitis infections [15].

Although studies have been done on co-infection seroprevalence in Ethiopia, limited data are available on the effect of co-infection on the immune status, liver function, and renal function. With this background, the present study was undertaken to assess the magnitude of HBV and HCV infection and its association with liver function, renal function and CD4+ T cell level of adult HAART taking patients at Zewditu Memorial Hospital, a model ART center in Addis Ababa, Ethiopia.

'Corresponding author: Nebiyou Yemanebrhane, Bethel Teaching General Hospital, Addis Ababa, Ethiopia, Tel: +2510910089081; E-mail: nebilyma@gmail.com

Received April 19, 2017; Accepted June 09, 2017; Published June 16, 2017

Citation: Yemanebrhane N, Addise D, Abebe N, Abebe F, Shewaamare A, et al (2017) Magnitude of Hepatitis B Virus and Hepatitis C Virus among HAART Taking Patients and Association with Liver and Renal Function and CD4+ T Cells Level. AIDS Clin Res 8: 702. doi: 10.4172/2155-6113.1000702

Copyright: (C) 2017 Yemanebrhane N, et al. This is an open-access article distributed under the terms of the Creative Commons Attribution License, which permits unrestricted use, distribution, and reproduction in any medium, provided the original author and source are credited. 
Citation: Yemanebrhane N, Addise D, Abebe N, Abebe F, Shewaamare A, et al. (2017) Magnitude of Hepatitis B Virus and Hepatitis C Virus among HAART Taking Patients and Association with Liver and Renal Function and CD4+ T Cells Level. J AIDS Clin Res 8: 702. doi: 10.4172/21556113.1000702

Page 2 of 7

\section{Materials and Methods}

Cross sectional study was conducted on 384 HAART taking study participants attending at Zewditu Memorial Hospital (ZMH) which is found in Addis Ababa, Ethiopia from January to June, 2016. The entire study participants were on HAART at least for one year. HIV/AIDS infected individuals who are taking HAART and adult ( $\geq 18$ years) were included while individuals who are on HAART but refused to give informed consent for additional testing of $\mathrm{HBV}$ and $\mathrm{HCV}$ were excluded. The hospital has the first and model ART center of the country. Sociodemographic information and other important possible risk factors of the study participants are routinely collected from the ART database and from their medical records using structured data collection sheet. Body mass index (BMI) of study participants were measured using the BMI formula. Based on their BMI study participants were grouped as underweight $\left(\mathrm{BMI}<18.5 \mathrm{~kg} / \mathrm{m}^{2}\right)$, Normal weight $\left(B M I \geq 18.5-25 \mathrm{~kg} / \mathrm{m}^{2}\right)$ and overweight $\left(25.0-29.9 \mathrm{~kg} / \mathrm{m}^{2}\right)$ and Obese $\left(\geq 30.0 \mathrm{~kg} / \mathrm{m}^{2}\right)$.

Eight $\mathrm{ml}$ of venous blood samples were aseptically collected using plain and EDTA vacutainer tubes ( $4 \mathrm{ml}$ in each tube) for the determination of HBV and HCV seroprevalence, CD4, renal function and liver function levels from each study participants. The blood specimen in the plain tube was centrifuged at 3000 RPM for $5 \mathrm{~min}$ to separate the serum and use for determination of liver function and renal function within one hour of separation. The remaining serum was kept in deep freezer $\left(-40^{\circ} \mathrm{C}\right)$ until detection of HBV and HCV. The second tube that contains whole blood was used for the CD4 levels determination. The CD4 count was determined by flow cytometry using BD FACScalibur (Becton Dickinson, San Jose, California). Liver and renal function tests were analyzed using Humastar 200 (Human $\mathrm{GmbH}$, Weisbaden, Germany). HBSAg and HCVAb were investigated by immunochromatographic technique (EUGENE cassette, Shangahai).

\section{Quality assurance}

All the laboratory analyses were performed following standard operating procedures. Both the CD4 count, Liver and renal function tests were carried out in nationally accredited laboratories $(\mathrm{ZMH}$, BGTH). Internal controls were run with each assay.

\section{Ethical considerations}

The study was conducted after being ethically cleared by Department of Medical Laboratory Sciences of Addis Ababa University and Addis Ababa Regional Health Bureau. Informed consent was obtained from the study participants after explaining the aim of the study including their right to withdraw from the study without compromising their care in the hospital. Confidentiality of data was maintained throughout the study.

\section{Statistical analysis}

Data was entered, cleaned and analyzed using SPSS version 20 software for windows. A descriptive analysis was done to determine mean and standard deviation for continuous variables and the difference in means was compared using independent-sample t-test. Statistical significance of categorical variable was evaluated by Chi-square test and p-value of less than 0.05 considered as statistically significant. Multivariate regression analyses to evaluate the role of confounding variables were performed.

\section{Results}

From the total of 384 study participants included in the study, 222 $(57.81 \%)$ were female and $162(42.19 \%)$ were male. Majority of study participants 168 (43.75\%) were in the age group of 30-39 years and 184 (47.92\%) were married as displayed in Table 1.

Overall prevalence of hepatitis $\mathrm{B}$ and $\mathrm{C}$ viruses among HAART taking study participants were 21 (5.7\%). Moreover HBV-HIV, HCV-HIV and HBV-HCV co-infection rates were 18 (4.7\%), 4 (1\%) and $1(0.3 \%)$, respectively as displayed in Table 2 . None of the socio-demographic variables shown in the table showed statistically significant association with HBV or HCV co-infection status (Table 2).

Liver and renal function tests of the participants was evaluated by measuring serum ALT, AST, ALP, Bilirubin total, total protein, albumin, urea, creatinine and uric acid. As shown in Table 3, none of the abnormalities noted for the various parameters tested significantly differ between HBV co-infected and HIV mono-infected participants ( $>0.05$ ). However, AST and ALT abnormality rates were higher among HCV-HIV co-infected patients compared to mono-infected ones $(\mathrm{p}<0.05)$.

Out of total study participants, $63.29 \%$ were having CD4 count below 500 cells $/ \mathrm{mm}^{3}$. Higher proportion of co-infected patients (71.43\%) compared to HIV mono-infected ones (62.83\%) had CD4 count below 500 cells $/ \mathrm{mm}^{3}$ after taking HAART for at least 1 year. Besides, $14.44 \%$ mono-infected and $23.81 \%(5 / 21)$ had CD4 count $<200$ (Figure 1). Chi square analysis showed no significant association between CD4 categories and co-infection status (Table 4).

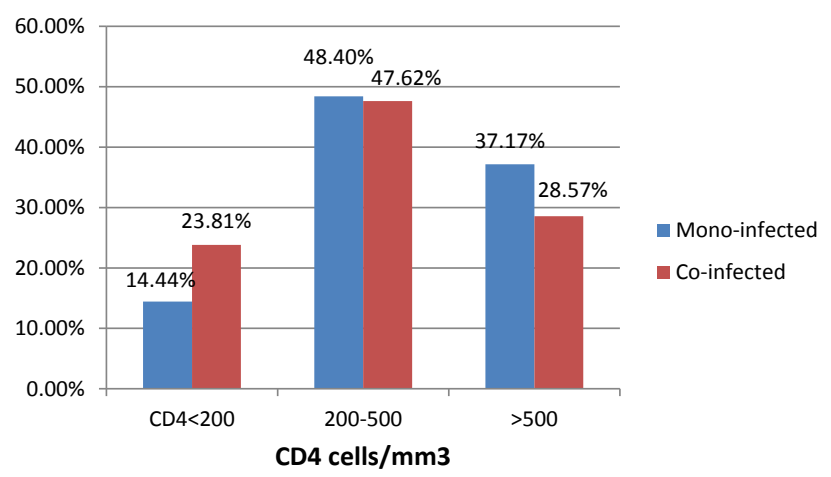

Figure 1: Distribution of mono-infected and co-infected HIV patients at Zewditu Memorial Hospital, Addis Ababa, Ethiopia, $2016(n=384)$.

\begin{tabular}{|c|c|c|c|c|}
\hline \multicolumn{2}{|l|}{ Variables } & \multicolumn{2}{|r|}{ Sex } & \multirow[b]{2}{*}{ Total N (\%) } \\
\hline & & Male N (\%) & Female $\mathbf{N}(\%)$ & \\
\hline \multirow[t]{4}{*}{ Age (year) } & $18-29$ & $21(5.47)$ & $40(10.42)$ & 61 (15.89) \\
\hline & $30-39$ & $47(12.24)$ & $121(31.51)$ & $168(43.75)$ \\
\hline & $40-49$ & $60(15.63)$ & 37 (9.64) & $97(25.26)$ \\
\hline & $\mathbf{2} \mathbf{5 0}$ & $34(8.85)$ & $24(6.25)$ & $58(15.10)$ \\
\hline \multirow{4}{*}{$\begin{array}{l}\text { Marital } \\
\text { status }\end{array}$} & Married & $100(26.04)$ & $84(21.88)$ & $184(47.92)$ \\
\hline & Divorced & $12(3.13)$ & 36 (9.37) & $48(12.5)$ \\
\hline & Not Married & 46 (11.98) & $56(14.58)$ & $102(26.56)$ \\
\hline & Widowed & $4(1.04)$ & $46(11.97)$ & $50(13.02)$ \\
\hline \multirow{5}{*}{$\begin{array}{l}\text { Education } \\
\text { status }\end{array}$} & Illiterate & $10(2.6)$ & $35(9.11)$ & 45 (11.72) \\
\hline & Elementary & $34(8.85)$ & 55 (14.32) & $89(23.18)$ \\
\hline & High school & $69(17.97)$ & $106(27.6)$ & 175 (45.57) \\
\hline & $\begin{array}{c}\text { Certificate and } \\
\text { above }\end{array}$ & $49(12.76)$ & $26(6.77)$ & 75 (19.53) \\
\hline & Total & $162(42.19)$ & $222(57.81)$ & $384(100 \%)$ \\
\hline
\end{tabular}

Table 1: Socio-demographic features of HAART taking study participants at Zewditu Memorial Hospital, Addis Ababa Ethiopia, $2016(n=384)$. 
Citation: Yemanebrhane N, Addise D, Abebe N, Abebe F, Shewaamare A, et al. (2017) Magnitude of Hepatitis B Virus and Hepatitis C Virus among HAART Taking Patients and Association with Liver and Renal Function and CD4+ T Cells Level. J AIDS Clin Res 8: 702. doi: 10.4172/21556113.1000702

Page 3 of 7

\begin{tabular}{|c|c|c|c|c|c|c|c|}
\hline \multicolumn{2}{|l|}{ Variables } & \multicolumn{2}{|c|}{ HBV-HIV } & \multirow[t]{2}{*}{$p$ value } & \multicolumn{2}{|c|}{ HCV-HIV } & \multirow[t]{2}{*}{ p value } \\
\hline & & \multirow{2}{*}{$\begin{array}{c}\text { Positive } \\
\text { N (\%) } \\
3(0.78)\end{array}$} & \multirow{2}{*}{$\begin{array}{c}\text { Negative } \\
\mathbf{N}(\%) \\
58(15.4)\end{array}$} & & $\begin{array}{c}\text { Positive } \\
\text { N (\%) }\end{array}$ & $\begin{array}{c}\text { Negative } \\
\mathbf{N}(\%)\end{array}$ & \\
\hline \multirow[t]{4}{*}{ Age } & $18-29$ & & & \multirow{4}{*}{0.102} & $0(0)$ & $61(15.9)$ & \multirow{4}{*}{0.175} \\
\hline & $30-39$ & $4(1.0)$ & $164(42.7)$ & & $2(0.5)$ & $166(43.22)$ & \\
\hline & $40-49$ & $5(1.3)$ & $92(23.95)$ & & $0(0)$ & $97(25.3)$ & \\
\hline & $\geq \mathbf{5 0}$ & $6(1.56)$ & $52(13.54)$ & & $2(0.5)$ & $56(14.56)$ & \\
\hline \multirow[t]{2}{*}{ Sex } & Male & $10(2.6)$ & $152(39.58$ & \multirow{2}{*}{0.239} & $2(0.5)$ & $160(41.67)$ & \multirow{2}{*}{0.750} \\
\hline & Female & $8(2.08)$ & $214(55.72)$ & & $2(0.5)$ & $220(57.29)$ & \\
\hline \multirow[t]{4}{*}{ BMI } & Underweight $\left(<18.5 \mathrm{~kg} / \mathrm{m}^{2}\right)$ & $3(0.78)$ & $54(14.06)$ & \multirow{4}{*}{0.777} & $2(0.5)$ & $55(14.32)$ & \multirow{4}{*}{0.211} \\
\hline & Normal $\left(18.5-24.9 \mathrm{~kg} / \mathrm{m}^{2}\right)$ & $10(2.6)$ & $204(53.12)$ & & $2(0.5)$ & $212(55.2)$ & \\
\hline & Overweight $\left(25.0-29.9 \mathrm{~kg} / \mathrm{m}^{2}\right)$ & $5(1.3)$ & $89(23.17)$ & & $0(0)$ & $94(24.47)$ & \\
\hline & Obese $\left(\geq 30.0 \mathrm{~kg} / \mathrm{m}^{2}\right)$ & $0(0)$ & $19(4.94)$ & & $0(0)$ & $19(4.94)$ & \\
\hline \multirow[t]{4}{*}{ Marital status } & Single & $5(1.3)$ & $97(25.26)$ & \multirow{4}{*}{0.481} & $0(0)$ & $102(26.6)$ & \multirow{4}{*}{0.450} \\
\hline & Married & $6(1.56)$ & $178(46.35)$ & & $3(0.78)$ & $181(47.13)$ & \\
\hline & Divorced & $4(1)$ & $44(11.45)$ & & $0(0)$ & $48(12.5)$ & \\
\hline & Widowed & $3(0.78)$ & $47(12.23)$ & & $1(0.2)$ & $49(12.76)$ & \\
\hline \multirow[t]{4}{*}{ Education status } & Illiterate & $1(0.2)$ & $44(11.45)$ & \multirow{4}{*}{0.674} & $1(0.2)$ & $44(11.45)$ & \multirow{4}{*}{0.387} \\
\hline & Elementary & $6(1.56)$ & $83(21.61)$ & & $2(0.5)$ & $87(22.65)$ & \\
\hline & High school & $8(2.08)$ & $167(43.48)$ & & $1(0.2)$ & $174(45.3)$ & \\
\hline & Certificate and above & $3(0.78)$ & $72(18.75)$ & & $0(0)$ & $75(19.53)$ & \\
\hline
\end{tabular}

BMI: Body Mass Index

Table 2: Distribution of HBV-HIV and HCV-HIV co-infections and its association with socio-demographic features of HAART taking study participants in Zewditu Memorial Hospital, Addis Ababa, Ethiopia, $2016(n=384)$.

\begin{tabular}{|c|c|c|c|c|c|c|c|}
\hline \multicolumn{2}{|c|}{ Liver and renal function tests } & \multicolumn{2}{|c|}{ HBV } & $p$ value & \multicolumn{2}{|c|}{$\mathrm{HCV}$} & p value \\
\hline AST & 0-34 IU/L & $\begin{array}{c}\text { Positive } \\
\mathbf{N} \\
13\end{array}$ & $\begin{array}{c}\begin{array}{c}\text { Negative } \\
\mathbf{N}\end{array} \\
274\end{array}$ & 0.801 & $\begin{array}{c}\text { Positive } \\
\mathbf{N} \\
0\end{array}$ & $\begin{array}{c}\begin{array}{c}\text { Negative } \\
\mathbf{N}\end{array} \\
287\end{array}$ & 0.001 \\
\hline & $>34$ IU/L & 5 & 92 & & 4 & 93 & \\
\hline \multirow[t]{2}{*}{ ALT } & 0-37 IU/L & 16 & 327 & \multirow{2}{*}{0.951} & 2 & 341 & \multirow[t]{2}{*}{0.010} \\
\hline & $>37$ IU/L & 2 & 39 & & 2 & 39 & \\
\hline \multirow[t]{2}{*}{ ALP } & 72-306 IU/L & 9 & 246 & \multirow[t]{2}{*}{0.131} & 3 & 252 & \multirow[t]{2}{*}{0.715} \\
\hline & $>306 \mathrm{IU} / \mathrm{L}$ & 9 & 120 & & 1 & 128 & \\
\hline \multirow[t]{2}{*}{ BILIRUBIN-T } & $0.2-0.9 \mathrm{mg} / \mathrm{dl}$ & 16 & 339 & \multirow[t]{2}{*}{0.558} & 4 & 351 & \multirow[t]{2}{*}{0.566} \\
\hline & $>0.9 \mathrm{mg} / \mathrm{dl}$ & 2 & 27 & & 0 & 29 & \\
\hline \multirow[t]{2}{*}{ T.PROTEIN } & $<6.6 \mathrm{~g} / \mathrm{dl}$ & 0 & 7 & \multirow[t]{2}{*}{0.636} & 0 & 7 & \multirow[t]{2}{*}{0.859} \\
\hline & $6.6-8.7 \mathrm{~g} / \mathrm{dl}$ & 18 & 359 & & 4 & 373 & \\
\hline \multirow[t]{2}{*}{ ALBUMIN } & $<3.5 \mathrm{~g} / \mathrm{dl}$ & 0 & 5 & \multirow[t]{2}{*}{0.8} & 0 & 5 & \multirow[t]{2}{*}{0.517} \\
\hline & $3.5-5.5 \mathrm{~g} / \mathrm{dl}$ & 18 & 361 & & 4 & 375 & \\
\hline \multirow[t]{2}{*}{ UREA } & $10-50 \mathrm{mg} / \mathrm{dl}$ & 18 & 360 & \multirow[t]{2}{*}{0.584} & 4 & 374 & \multirow[t]{2}{*}{0.8} \\
\hline & $>50 \mathrm{mg} / \mathrm{dl}$ & 0 & 6 & & 0 & 6 & \\
\hline \multirow[t]{2}{*}{ CREATININE } & $0.5-1.2 \mathrm{mg} / \mathrm{dl}$ & 12 & 229 & \multirow[t]{2}{*}{0.725} & 4 & 237 & \multirow[t]{2}{*}{0.121} \\
\hline & $>1.2 \mathrm{mg} / \mathrm{dl}$ & 6 & 137 & & 0 & 143 & \\
\hline \multirow[t]{2}{*}{ URICACID } & $<7.0 \mathrm{mg} / \mathrm{dl}$ & 15 & 302 & \multirow[t]{2}{*}{0.929} & 3 & 314 & \multirow[t]{2}{*}{0.689} \\
\hline & $>7.0 \mathrm{mg} / \mathrm{dl}$ & 3 & 64 & & 1 & 66 & \\
\hline
\end{tabular}

AST: Aspartate Amino Transaminase; ALT: Alanine Amino Transaminase; ALP: Alkaline Phosphatase

Table 3: Comparisons of liver and renal function tests abnormalities between HIV mono-infected and HBV/HCV co-infected HAART taking patients at Zewditu Memorial Hospital, Addis Ababa, Ethiopia, $2016(n=384)$.

\begin{tabular}{|c|c|c|c|c|c|c|c|}
\hline \multirow[t]{2}{*}{ Variable } & \multicolumn{3}{|c|}{ HBV-HIV } & \multirow[t]{2}{*}{$P$ value } & \multicolumn{2}{|c|}{ HCV-HIV } & \multirow[t]{2}{*}{$P$ value } \\
\hline & \multicolumn{2}{|c|}{$\begin{array}{c}\text { Yes } \\
\text { N (\%) }\end{array}$} & $\begin{array}{c}\text { No } \\
\text { N (\%) }\end{array}$ & & $\begin{array}{c}\text { Yes } \\
\text { N (\%) }\end{array}$ & $\begin{array}{c}\text { No } \\
\text { N (\%) }\end{array}$ & \\
\hline \multirow[t]{2}{*}{ CD4 Cells/ $\mu \mathrm{L}$} & Normal & $5(1.30)$ & $136(35.41)$ & \multirow{2}{*}{0.420} & $1(0.26)$ & $140(36.45)$ & \multirow[b]{2}{*}{0.625} \\
\hline & Abnormal & $13(3.38)$ & $230(59.89)$ & & $3(0.78)$ & 240 (62.5) & \\
\hline
\end{tabular}

Table 4: CD4 level and its association with co-infection in Zewditu Memorial Hospital, Addis Ababa, Ethiopia, 2016.

The mean \pm SD levels of liver function tests, renal function tests and CD4 count by co-infection status are displayed in Table 5. Except for renal function tests, the mean values were higher for liver function tests and lower for CD4 counts in co-infected participants. However, the difference reached a statistical significant level for AST $(p=0.016)$ and marginally significant for ALP ( $\mathrm{p}=0.072)$ and total protein $(\mathrm{p}=0.063)$. 
Citation: Yemanebrhane N, Addise D, Abebe N, Abebe F, Shewaamare A, et al. (2017) Magnitude of Hepatitis B Virus and Hepatitis C Virus among HAART Taking Patients and Association with Liver and Renal Function and CD4+ T Cells Level. J AIDS Clin Res 8: 702. doi: 10.4172/21556113.1000702

Page 4 of 7

\begin{tabular}{|c|c|c|c|c|}
\hline \multicolumn{2}{|l|}{ Parameters } & $\mathbf{N}$ & Mean \pm SD & p-value \\
\hline \multirow[t]{2}{*}{ AST (IU/L) } & mono infected & 363 & $28.06 \pm 14.13$ & \multirow[t]{2}{*}{0.016} \\
\hline & co-infected & 21 & $35.95 \pm 20.44$ & \\
\hline \multirow[t]{2}{*}{ ALT (IU/L) } & mono infected & 363 & $19.72 \pm 11.40$ & \multirow[t]{2}{*}{0.457} \\
\hline & co-infected & 21 & $21.61 \pm 10.14$ & \\
\hline \multirow[t]{2}{*}{ ALP (IU/L) } & mono infected & 363 & $286.98 \pm 139.17$ & \multirow[t]{2}{*}{0.072} \\
\hline & co-infected & 21 & $344.47 \pm 187.05$ & \\
\hline \multirow[t]{2}{*}{ UREA (mg/dl) } & mono infected & 363 & $23.63 \pm 16.80$ & \multirow[t]{2}{*}{0.564} \\
\hline & co-infected & 21 & $23.63 \pm 8.44$ & \\
\hline \multirow[t]{2}{*}{ CREAT (mg/dl) } & mono infected & 363 & $1.0 \pm 2.13$ & \multirow[t]{2}{*}{0.532} \\
\hline & co-infected & 21 & $0.75 \pm 0.23$ & \\
\hline \multirow[t]{2}{*}{ URICACID(mg/dl) } & mono infected & 363 & $4.42 \pm 1.42$ & \multirow[t]{2}{*}{0.940} \\
\hline & co-infected & 21 & $4.44 \pm 1.37$ & \\
\hline \multirow[t]{2}{*}{ BIL.T(mg/dl) } & mono infected & 363 & $0.95 \pm 4.70$ & \multirow[t]{2}{*}{0.820} \\
\hline & co-infected & 21 & $0.72 \pm 0.61$ & \\
\hline \multirow[t]{2}{*}{ T.PROT(g/dl) } & mono infected & 363 & $8.42 \pm 1.07$ & \multirow[t]{2}{*}{0.063} \\
\hline & co-infected & 21 & $8.87 \pm 1.39$ & \\
\hline \multirow[t]{2}{*}{ ALBUMIN(g/dl) } & mono infected & 363 & $7.09 \pm 0.68$ & \multirow[t]{2}{*}{0.793} \\
\hline & co-infected & 21 & $5.22 \pm 0.65$ & \\
\hline \multirow[t]{2}{*}{ 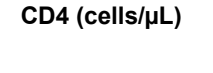 } & mono infected & 363 & $449.31 \pm 232.85$ & \multirow[t]{2}{*}{0.374} \\
\hline & co-infected & 21 & $402.76 \pm 233.87$ & \\
\hline
\end{tabular}

AST: Aspartate Amino Transaminase; ALT: Alanine Amino Transaminase; ALP: Alkaline Phosphatase; CD4: Cluster of Differentiation 4

Table 5: Mean level of biochemical parameter and association with co-infection in ART clinic of Zewditu Memorial Hospital, Addis Ababa, 2016.

When analyzed by hepatitis virus types, serum AST $(67.25+17.23$ vs $28.06 \pm 14.13, \mathrm{p}=0.0001)$ and ALT $(33.25+10.37$ vs. $19.72 \pm 11.40$, $\mathrm{p}=0.017)$ were significantly higher in HCV-HIV co-infected patients and serum ALP $(354.83+198.84$ vs. $286.95+138.74, p=0.048)$ was significantly higher in HBV-HIV co- infected patients compared to the mono-infected ones. Age and CD4 count were not significantly associated with abnormal level of liver and renal function parameters of the study ( $\mathrm{p}$ value $>0.05$ ). Multivariate regressions were run to predict liver and renal function parameters from the independent variables studied in this study. Patient Sex was statistically significantly predict AST (0.022) and Uric acid (0.001) level while patient BMI was statistically significantly predict Uric acid (0.014) level. Majority of study participant were in $>200$ cells $/ \mathrm{mm}^{3}$ counts and similarly total protein and albumin level of study participant were in normal status (Table 6).

\section{Discussion}

$\mathrm{HBV}$ and $\mathrm{HCV}$ are the major cause of chronic liver disease worldwide and they share similar route of transmission with HIV. As a result, HIV positive individuals are at risk for co-infection either with HBV or HCV. Co-infection either with HBV or HCV increase the risk of HAART related hepatotoxicity. Hence, this study investigated the magnitude of HBV and HCV infection among adult HAART taking patients and its association with liver function, renal function and CD4+ T cell levels.

The overall burden of HBV and HCV among the HIV infected patients who were on HAART for at least 1 year in this study was $5.7 \%$ which is lower than studies in Northwest Ethiopia (11.7\%), Kampala (22.5\%), Cambodia (16.3\%), Kenya (15.3\%) and Vietnam (50.3\%) [1619]. The finding is close to what has been reported from Addis Ababa (6.0\%) by Abera et al. [20]. The observed 4.7\% HIV-HBV co-infection rate was lower compared with the study in North West Ethiopia which reported 5.6\% [12]. Conversely, HIV-HBV co-infection rate of this study was higher as compared to worldwide HBV prevalence which was $3.1 \%$ [21] and also to studies by Manyazewal et al. (2.8\%) and Shimles et al. (3.9\%) [22,23], both from Addis Ababa and lower than study in Kenya (6\%) and Nigeria (6\%) $[19,24]$. The reason for such variations in co-infection rate among HIV infected patients could possibly be due to difference in the distribution of these viruses in the various geographic locations, recruitment setting, sample size and ethnic variation. In this study the prevalence of HIV-HBV co-infection was slightly higher in males than females $(2.6 \%$ vs. $2.08 \%)$ which also resembles with study by Wondimeneh et al. [12].

The seroprevalence of HIV-HCV co-infection in this study was 1.0 $\%$ which is lower than World Health Organization estimate of HCV infection in general population(3\%) [25], in south India (8.3\%), Nigeria $(4.0 \%)$ and Ethiopia $(5.0 \%)[12,26,27]$. On the other hand in this study non statistically significant higher prevalence of HCV-HIV co-infection was also observed in married $(0.78 \%)$ which support a study in North West Gondar by Wondimeneh et al. [12]. The reason for the HCV variation in HIV infected individuals in the different studies might share the factors responsible for HBV prevalence variation discussed above.

This study indicates that co-infection of HBV and HCV were uncommon with $1(0.2 \%)$ person infected with both viruses. Similar findings have been reported in Nigeria [28] and Bangkok [29] where the prevalence of $\mathrm{HBV} / \mathrm{HCV}$ co-infection was $0.4 \%$. However, the finding of this study was lower when compared to studies in Vietnam (6.5\%), Kenya (1\%) and northern Ethiopia (1\%) $[12,19,20]$. Risk factors which accounts for HBV and HCV prevalence difference might work for the triple infection variation as well.

Biochemical laboratory tests are of immense value in diagnosis and monitoring of liver disease. Regarding liver enzymes, in this study there was a significant increase in the mean level of serum ALT and AST in HCV-HIV co-infected patients $(\mathrm{p}<0.05)$ compared to HIV monoinfected ones. Similarly the mean level of serum AST and ALP were significantly higher in co-infected patients. This supports other studies conducted by Olawumi et al., Abera et al., who reported a high mean level of ALT among HIV patients co-infected either with HBV or HCV 
Citation: Yemanebrhane N, Addise D, Abebe N, Abebe F, Shewaamare A, et al. (2017) Magnitude of Hepatitis B Virus and Hepatitis C Virus among HAART Taking Patients and Association with Liver and Renal Function and CD4+ T Cells Level. J AIDS Clin Res 8: 702. doi: 10.4172/21556113.1000702

Page 5 of 7

\begin{tabular}{|c|c|c|c|c|c|c|c|c|c|c|c|c|c|c|c|c|}
\hline \multicolumn{2}{|c|}{ VARIABLE } & \multicolumn{2}{|c|}{ AST } & \multirow[t]{2}{*}{$p$} & \multicolumn{2}{|c|}{ ALT } & \multirow[t]{2}{*}{$p$} & \multicolumn{2}{|c|}{ ALP } & \multirow[t]{2}{*}{ p } & \multicolumn{2}{|c|}{ Urea } & \multirow[t]{2}{*}{$p$} & \multicolumn{2}{|c|}{ Creatinine } & \multirow[t]{2}{*}{$\mathbf{p}$} \\
\hline & & (IU/L) & -34 (IUIL) & & (IVIL) & (IU/L) & & $\begin{array}{c}306 \\
(\mathrm{IU} / \mathrm{L})\end{array}$ & (IU/L) & & (mg/dl) & $\begin{array}{l}(\mathrm{mg} / \\
\mathrm{dl})\end{array}$ & & (mg/dl) & (mg/dl) & \\
\hline \multirow[t]{4}{*}{ age } & $18-29$ & 47 & 14 & \multirow[t]{4}{*}{0.745} & 54 & 7 & \multirow[t]{4}{*}{0.495} & 45 & 16 & \multirow[t]{4}{*}{0.242} & 39 & 2 & \multirow[t]{4}{*}{0.394} & 31 & 30 & \multirow[t]{4}{*}{0.867} \\
\hline & $30-39$ & 126 & 42 & & 152 & 16 & & 117 & 51 & & 167 & 1 & & 114 & 54 & \\
\hline & $40-49$ & 70 & 27 & & 87 & 10 & & 57 & 40 & & 96 & 1 & & 65 & 32 & \\
\hline & $\geq \mathbf{5 0}$ & 44 & 14 & & 50 & 8 & & 36 & 22 & & 56 & 2 & & 31 & 27 & \\
\hline \multirow[t]{2}{*}{$\operatorname{sex}$} & $F$ & 172 & 50 & \multirow[t]{2}{*}{$0.022^{*}$} & 202 & 20 & \multirow[t]{2}{*}{0.327} & 149 & 73 & \multirow[t]{2}{*}{0.729} & 220 & 2 & \multirow[t]{2}{*}{0.885} & 143 & 79 & \multirow[t]{2}{*}{0.965} \\
\hline & M & 115 & 47 & & 141 & 21 & & 106 & 56 & & 158 & 4 & & 98 & 64 & \\
\hline \multirow[t]{4}{*}{ BMI } & $\begin{array}{l}\text { Underweight } \\
\left(<18.5 \mathrm{~kg} / \mathrm{m}^{2}\right)\end{array}$ & 42 & 15 & \multirow[t]{4}{*}{0.305} & 53 & 4 & \multirow[t]{4}{*}{0.469} & 32 & 25 & 0.858 & 56 & 1 & 0.566 & 34 & 23 & 0.472 \\
\hline & $\begin{array}{c}\text { Normal }(18.5-24.9 \\
\left.\mathrm{kg} / \mathrm{m}^{2}\right)\end{array}$ & 162 & 52 & & 194 & 20 & & 149 & 65 & & 210 & 4 & & 134 & 80 & \\
\hline & $\begin{array}{c}\text { Overweight } \\
\left(25.0-29.9 \mathrm{~kg} / \mathrm{m}^{2}\right)\end{array}$ & 69 & 25 & & 81 & 13 & & 61 & 33 & & 93 & 1 & & 61 & 33 & \\
\hline & $\begin{array}{c}\text { Obese }(\geq 30.0 \\
\left.\mathrm{kg} / \mathrm{m}^{2}\right)\end{array}$ & 14 & 5 & & 15 & 4 & & 13 & 6 & & 19 & 0 & & 12 & 7 & \\
\hline CD4 & $<200$ cells $/ \mathrm{mm}^{3}$ & 41 & 18 & 0.926 & 52 & 7 & 0.314 & 36 & 23 & 0.099 & 58 & 1 & 0.060 & 36 & 23 & 0.229 \\
\hline & $>200$ cells $/ \mathrm{mm}^{3}$ & 246 & 79 & & 291 & 34 & & 219 & 106 & & 320 & 5 & & 205 & 120 & \\
\hline & VARIABLE & Uric & acid & p & Bilirub & in $T$ & p & T.pro & tein & p & Albun & $\operatorname{nin}$ & p & & & \\
\hline & & $\begin{array}{l}<7.0(\mathrm{mg} / \\
\text { dl) }\end{array}$ & $\begin{array}{c}>7.0(\mathrm{mg} / \\
\mathrm{dl})\end{array}$ & & $\begin{array}{l}0.2-0.9 \\
(\mathrm{mg} / \mathrm{dl})\end{array}$ & $\begin{array}{c}>0.9 \\
(\mathrm{mg} / \mathrm{dl})\end{array}$ & & $\begin{array}{c}6.6- \\
8.7(\mathrm{~g} / \\
\mathrm{dl})\end{array}$ & $\begin{array}{l}<6.6 \\
(g / d l)\end{array}$ & & $\begin{array}{c}3.5-5.5 \\
(\mathrm{~g} / \mathrm{dl})\end{array}$ & $\begin{array}{l}<3.5 \\
(g / \\
\text { dl) }\end{array}$ & & & & \\
\hline Age & $18-29$ & 52 & 9 & 0.413 & 59 & 2 & 0.903 & 61 & 0 & 0.964 & $59-$ & 2 & 0.99 & & & \\
\hline & $30-39$ & 137 & 31 & & 157 & 11 & & 163 & 5 & & 166 & 2 & & & & \\
\hline & $40-49$ & 78 & 19 & & 86 & 11 & & 95 & 2 & & 97 & 0 & & & & \\
\hline & $\geq 50$ & 50 & 8 & & 53 & 5 & & 58 & 0 & & 57 & 1 & & & & \\
\hline Sex & $\mathbf{F}$ & 204 & 18 & $0.001^{*}$ & 209 & 13 & 0.846 & 218 & 4 & 0.145 & 220 & 2 & 0.969 & & & \\
\hline & M & 113 & 49 & & 146 & 16 & & 159 & 3 & & 159 & 3 & & & & \\
\hline BMI & $\begin{array}{l}\text { Underweight } \\
\left(<18.5 \mathrm{~kg} / \mathrm{m}^{2}\right)\end{array}$ & 50 & 7 & $0.014^{*}$ & 54 & 3 & 0.860 & 57 & 0 & 0.302 & 56 & 1 & 0.986 & & & \\
\hline & $\begin{array}{c}\text { Normal }(18.5-24.9 \\
\left.\mathrm{kg} / \mathrm{m}^{2}\right)\end{array}$ & 177 & 37 & & 198 & 16 & & 207 & 7 & & 211 & 3 & & & & \\
\hline & $\begin{array}{c}\text { Overweight } \\
\left(25.0-29.9 \mathrm{~kg} / \mathrm{m}^{2}\right)\end{array}$ & 76 & 18 & & 86 & 8 & & 94 & 0 & & 93 & 1 & & & & \\
\hline & $\begin{array}{c}\text { Obese }(\geq 30.0 \\
\left.\mathrm{kg} / \mathrm{m}^{2}\right)\end{array}$ & 14 & 5 & & 17 & 2 & & 19 & 0 & & 19 & 0 & & & & \\
\hline CD4 & $<200$ cells $/ \mathrm{mm}^{3}$ & 45 & 14 & 0.066 & 52 & 7 & 0.290 & 59 & 0 & 0.560 & 58 & 1 & 0.218 & & & \\
\hline count & $>200$ cells $/ \mathrm{mm}^{3}$ & 272 & 53 & & 303 & 22 & & 318 & 7 & & 321 & 4 & & & & \\
\hline
\end{tabular}

AST: Aspartate Amino Transaminase; ALT: Alanine Amino Transaminase; ALP: Alkaline Phosphatase; CD4: Cluster of Differentiation 4; BMI: Body Mass Index

Table 6: Association among age, sex, BMI, CD4 cell count, renal function and liver function tests in patients with HIV receiving HAART at Zewditu Memorial Hospital Addis Ababa, Ethiopia in 2016.

$[16,23]$. Moreover this study strongly supported the study conducted in Northwest Ethiopia which reported a raised level of liver enzyme in coinfected HIV patients even though it is not statistically significant [12].

In this study the highest increment in the liver enzymes ALT and AST was observed among HIV patients co-infected with HCV compared to HBV co-infected ones. This might be as a result of HCVs' nature to cause chronic viral hepatitis that can lead to cirrhosis and hepatocellular carcinoma [30]. In addition these enzymes level further increment in case of HCV-HIV co-infection could be due to viral hepatitis infection as well as patient's condition like having chronic alcoholism or due to drug induced hepatotoxicity.

On the other hand markers for liver dysfunction (bilirubin total, total protein and albumin) were not statistically significantly different among patient with HIV mono-infected and co-infected with either HBV or HCV. This is similar to the study conducted in Nigeria by Olawumi et al. [24]. On contrary a study in southern India by Chandra et al. reported that co-infected HIV patients have statistically different liver dysfunction markers compared to mono-infected HIV patients [26] in this study statistically low level of albumin and total protein was not seen. This might be due to the nature of total protein and albumin tends to be normal in disease like acute viral hepatitis and drug related hepatotoxicity.

According to this study renal function tests (urea, creatinine, uric acid) were not statistically significant among co and mono-infected HIV patients which supports the study conducted in Nigeria by Otegbayo et al. who reported that serum urea and creatinine levels between HBV-HIV and HIV only infected patient were not statistically significant $(p>0.05)$ [31].

In general, in this study there was no significant increment of mean CD4+ T cells value of HIV mono-infected patients when compared to co-infected patients. Despite the absence of statistical significance in the mean level of CD4+ T cells between HIV-mono-infected and HIV-viral hepatitis co-infected individuals, lower mean level of CD4 were found in co-infected individuals. Which were similar to that of the study in Gondar, northern Ethiopia [12]. 
Citation: Yemanebrhane N, Addise D, Abebe N, Abebe F, Shewaamare A, et al. (2017) Magnitude of Hepatitis B Virus and Hepatitis C Virus among HAART Taking Patients and Association with Liver and Renal Function and CD4+ T Cells Level. J AIDS Clin Res 8: 702. doi: 10.4172/21556113.1000702

Page 6 of 7

The absolute $\mathrm{CD} 4+$ count is an important predictive bio-marker that can be employed in establishing decision points for initiating appropriate therapy as well as monitoring in HIV positive patients in resource limited settings. In the present study HBV-HIV co-infected patients had lower mean level of CD4 compared to mono-infected ones which is intensely supported by Wondimeneh et al., Olawumi et al., Chandra et al. and Griensven et al. [12,18,24,26]. This might be due to HBV's nature to lead severe liver disease including acute hepatitis cirrhosis, and hepatocellular carcinoma. In addition successful clearance of the virus as well as the establishment of liver disease is largely driven by a complex interaction between the virus and host immune response.

Similarly, HCV-HIV co-infected patient have lower mean level of CD4 compared to mono-infected ones. This outcome is supported by studies conducted by several studies $[12,18,24,26]$ this could be a confirmation for HCV infection character by the presence of functionally and phenotypically altered $\mathrm{T}$ cell responses (low CD4 count) that are unable to clear the virus but most likely contribute to the ongoing liver disease.

Though there was only one triple infected patient in the current study (HBV-HCV-HIV), his CD 4 count 151 cells/ $\mu \mathrm{L}$ was remarkably lower than the average count for mono- as well as co-infected patients. The finding in this patient is consistent with the study conducted by Wondimenh et al. and Forbi et al. who reported that patients co-infected with HIV/HBV/ $\mathrm{HCV}$ appeared to have lower CD4+ count compared to HBV/HIV-only, $\mathrm{HCV} / \mathrm{HIV}$-only and patients with mono HIV infection [12,32]. This might be due to the fact that HBV/HCV/HIV triple infection raises the chance of virological and immunological failure.

\section{Conclusion}

The present study shows that prevalence of HBV and HCV among HAART taking HIV patients are lower than most of the previous studies. Moreover, this study indicates that, $\mathrm{HBV}$ and $\mathrm{HCV}$ co-infected HIV positive patients are more likely to have abnormal liver enzyme test result than mono-infected HIV patients though reasonably similar renal function test result was observed. On the other hand, mean CD4 count was lower among co-infected HIV patients. Of interest, remarkable proportion of co-infected patients after 1 year of HAART still have CD4 count $<200$ cells/ $\mu \mathrm{L}$. Finally, further large scale and cohort studies should be performed in order to observe the effect of co-infection on HAART taking patients.

\section{Limitations and Constraints}

The measurement, HIV viral load, HBV DNA and HCV RNA viremia assays were required but these tests were not done due financial limitations.

\section{Authors' Contribution and Information}

Nebiyou Yemanebrhane: Design the project, perform the laboratory work and prepare the manuscript

Aster Tsegaye: Main advisor of the project

Aster Shewaamare: Clinical supervisor

Desalegn Adisse: Prepare the manuscript

Nardos Abebe: Prepare the manuscript

Fassika Abebe: Prepare the manuscript

All authors contributed to the preparation, revision and final approval of the manuscript.

\section{Acknowledgement}

We gratefully acknowledge the study participants, Zewditu Memorial Hospital Addis Ababa Regional Laboratory and Bethel Teaching General Hospital Medica laboratory colleagues and staffs, Addis Ababa, Ethiopia.

\section{References}

1. Ganem D, Schneider RJ (2001) Hepadnaviridae and their replication. Fields Virology, Lippincott-Raven Publishers, Philadelphia, PA 4

2. Kremsdorf D, Soussan P, Paterlini-Brechot P, Brechot C (2006) Hepatitis virus-related hepatocellular carcinoma: Paradigms for viral-related human carcinogenesis. Oncogene 25: 3823-3833.

3. Choo QL, Kuo G, Weiner AJ, Overby LR, Bradley DW, et al. (1989) Isolation of a cDNA clone derived from a blood-borne non-A, non-B viral hepatitis genome. Science 244: 359-362

4. Barre-Sinoussi F (1996) HIV as the cause of AIDS. Lancet 348: 31-35

5. Joint United Nations Programme on HIVIAIDS (UNAIDS) (2013) UNAIDS report on the global AIDS epidemic.

6. Alter MJ (2003) Epidemiology of hepatitis B in Europe and worldwide. J Hepato 39: S64-S69.

7. Alter MJ (2006) Epidemiology of viral hepatitis and HIV co-infection. J Hepato 44: S6-S9.

8. Chu CJ, Lee SD (2008) Hepatitis B virus/hepatitis C virus co-infection: Epidemiology, clinical features, viral interactions and treatment. J Gastroenterol Hepatol 23: 512-520

9. Belyhun Y, Maier M, Mulu A, Diro E, Liebert UG (2016) Hepatitis viruses in Ethiopia: A systematic review and meta-analysis. BMC Infect Dis 16: 761.

10. Barth RE, Huijgen Q, Taljaard J, Hoepelman AIM (2010) Hepatitis B/C and HIV in sub-Saharan Africa: An association between highly prevalent infectious diseases - A systematic review and meta-analysis. Int $\mathrm{J}$ Infect Dis 14 e1024-e1031.

11. http://files.unaids.org/en/dataanalysis/knowyourresponse/countryprogressrepo rts/2012countries/GAP Report 2012.pdf

12. Wondimeneh Y, Alem M, Asfaw F, Belyhun Y (2013) HBV and HCV seroprevalence and their correlation with CD4 cells and liver enzymes among HIV positive individuals at University of Gondar Teaching Hospital, Northwest Ethiopia. Virol J 10: 1-8.

13. Nguen TA, Sterling RK (2013) Viral hepatitis B co-infection with human immunodeficiency virus, hepatitis D virus or hepatitis $C$ virus. Clin Liver Dis 2: $45-48$.

14. Kim AY, Chung RT, Polsky B (2001) Human immunodeficiency virus and hepatitis $B$ and $C$ coinfection: Pathogenic interactions, natural history and therapy. AIDS Clin Rev 2001: 263-306.

15. Adewole OO, Anteyi E, Ajuwon Z, Wada I, Elegba F, et al. (2009) Hepatitis B and $C$ virus co-infection in Nigerian patients with HIV infection. J Infect Dev Ctries 3: 369-375

16. Baseke J, Musenero M, Mayanja-Kizza H (2015) Prevalence of hepatitis B and $C$ and relationship to liver damage in HIV infected patients attending Join Clinical Research Centre Clinic (JCRC), Kampala, Uganda. Afr Health Sci 15: 322-327.

17. Griensven J, Phirum L, Choun K, Thai $S$,Weggheleire AD, et al. (2014) Hepatitis $B$ and $C$ co-infection among HIV-infected adults while on Antiretroviral treatment: Long-term survival, CD4 cell count recovery and antiretroviral toxicity in Cambodia. PLoS ONE 9: 2.

18. Muriuki BM, Gicheru MM, Wachira D, Nyamache AK, Khamadi SA (2013) Prevalence of hepatitis $B$ and $C$ viral co-infections among HIV-1 infected individuals in Nairobi, Kenya. BMC Res Notes 6: 363.

19. Huy BV, Vernavong K, Kính NV (2014) HBV and HCV co-infection among HIVI AIDS patients in the national hospital of tropical diseases, Vietnam. AIDS Res Treat 2014: 581021.

20. Abera B, Zenebe Y, Mulu W, Kibret M, Kahsu G (2014) Seroprevalence of hepatitis $B$ and $C$ viruses and risk factors in HIV infected children at the Felgehiwot Referral Hospital, Ethiopia. BMC Res Notes 7: 838.

21. Schweitzer A, Horn J, Mikolajczyk RT, Krause G, Ott JJ (2015) Estimations of 
Citation: Yemanebrhane N, Addise D, Abebe N, Abebe F, Shewaamare A, et al. (2017) Magnitude of Hepatitis B Virus and Hepatitis C Virus among HAART Taking Patients and Association with Liver and Renal Function and CD4+ T Cells Level. J AIDS Clin Res 8: 702. doi: 10.4172/21556113.1000702

Page 7 of 7

worldwide prevalence of chronic hepatitis B virus infection: A systematic review of data published between 1965 and 2013. Lancet 386: 1546-1555.

22. Manyazewal T, Sisay Z, Biadgilign S, Abegaz WE (2014) Hepatitis B and hepatitis $C$ virus infections among antiretroviral-naive and -experienced HIV co-infected adults. J Med Microbiol 63: 742-747.

23. Shimeis T, Torben W, Medhin G, Tebeje M, Andualem A, et al. (2008) Hepatitis $B$ virus infection among people attending the voluntary counseling and testing centre and anti-retroviral therapy clinic of St Paul's General Specialized Hospital, Addis Ababa, Ethiopia. Sex Transm Infect 84: 37-41.

24. Olawumi H, Olanrewaju D, Shittu A, Durotoye I, Akande A, et al. (2014) Effect of hepatitis-B virus co-infection on CD4 cell count and liver function of HIV infected patients. Ghana Med J 48: 96-100.

25. Liu Z, Hou J (2006) Hepatitis B virus (HBV) and hepatitis C virus (HCV) dual infection. Int J Med Sci 3: 57-62.

26. Chandra N, Joshi N, Raju YSN, Kumar A, Teja VD (2013) Hepatitis B and/or C co-infection in HIV infected patients: A study in a tertiary care center from south India. Indian J Med Res 138: 950-954.

27. Kennedy IC, Yibala O (2014) Patterns of CD4+ cells and liver enzymes and their correlation with prevalence of $\mathrm{HBV}$ and $\mathrm{HCV}$ among HIV positive individuals at
Orlu Hospital counseling and testing unit, Imo state, South-East Nigeria. Int J Curr Microbiol App Sci 3: 296-303.

28. Okwuraiwe A, Audu R, Salu O, Onwuamah C, Amoo O, et al. (2013) Immunological and virological response to HAART in HIV-1 patients co-infected with hepatitis B and C viruses. W Afr J Med 31: 124-128.

29. Tankhiwale SS, Khadase RK, Jalgoankar SV (2003) Seroprevalence of anti$\mathrm{HCV}$ and hepatitis B surface antigen in HIV infected patients. Ind $\mathrm{J} \mathrm{Med}$ Microbiol 21: 268-270.

30. Sulkowski MS, Mast E, Seeff LB, Thomas DL (2000) Hepatitis C virus infection as an opportunistic disease in persons infected with human immunodeficiency virus. Clin Infect Dis 30: S77-S84.

31. Otegbayo JA, Akingbola TS, Akinyemi JO, Adedapo KS, Odaibo GN, et al. (2011) Immunovirological and biochemical changes in nigerian patients with hepatitis B co-infection on antiretroviral therapy. World J AIDS 1: 31-36.

32. Forbi J, Gabadi S, Alabi R, Iperepolu H, Pam C, et al. (2007) The role of triple infection with hepatitis $B$ virus, hepatitis $C$ virus and human immunodeficiency virus (HIV) type-1 on CD4+ lymphocyte levels in the highly HIV infected population of North-Central Nigeria. Memórias do Instituto Oswaldo Cruz 102 535-537. 